\title{
Effect of Eight Weeks of Exercise Training on Lipid Profile and Insulin Sensitivity in Obese Person
}

\author{
NGAYIMBESHA Adrien", BIZIMANA Jean Berchmans and GAKIMA Marie Stella
}

Institute of Physical Education and Sports, University of Burundi, Burundi

*Corresponding author: NGAYIMBESHA Adrien, Institute of Physical Education and Sports, University of Burundi, Burundi

\section{Introduction}

Obesity is a metabolic abnormality characterized by increased levels of plasma free fatty acids and triglycerides, decreased levels of High-Density Lipoprotein (HDL), and abnormal Low-Density Lipoprotein (LDL) composition [1]. The most significant contributing factor for obesity-related dyslipidemia is likely uncontrolled fatty acid release from adipose tissue, especially visceral adipose tissue, through lipolysis, which causes increased delivery of fatty acids to the liver and synthesis of Very-Low-Density Lipoprotein (VLDL). Increased levels of free fatty acids can decrease mRNA expression or activity of Lipoprotein Lipase (LPL) in adipose tissue and skeletal muscle, and increased synthesis of VLDL in the liver can inhibit lipolysis of chylomicrons, which promotes hypertriglyceridemia [2].

Another metabolic abnormality associated to obesity and increased body lipids is insulin resistance. Insulin Resistance (IR) is defined as a defective metabolic response of insulin to stimulate glucose uptake into skeletal muscle and adipose tissue and/or to suppress hepatic gluconeogenesis and glucose release into circulation [3]. The metabolic response of insulin resistance and subsequent hyperinsulinemia is attributed to the development of serious health consequences such as overweight, hypertension, hyperlipidemia, cardiovascular disease, and type-2 diabetes [3]. But, how is pathophysiology of dyslipidemia associated with insulin resistance? Altered metabolism of TG-rich lipoproteins plays a pivotal role in the pathophysiology of dyslipidemia associated with insulin resistance. Alterations include increased hepatic secretion, as well as impaired clearance of VLDL and intestinally derived chylomicrons [4]. An important consequence of slow clearance is the persistence in the circulation of VLDL, postprandial chylomicrons and partially metabolized remnant particles. Remnants include cholesterol-enriched intermediate-density lipoproteins that have been shown to confer high atherogenic risk in experimental animal models as well as in man [5]. Overproduction of VLDL, with increased secretion of TG and ApoB100, appears to be central in the etiology of increased plasma VLDL levels in patients with insulin resistance or T2DM [5]. Impairment of muscle glucose uptake and oxidation is a consequence of insulin resistance in muscle, while impairment of hepatic glucose production is a consequence of insulin resistance in liver. Finally, resistance to insulin's suppressive action in adipose tissue is accounting for the increased lipolysis in the insulin-resistant individual. Other research showed steps by which excess lipid depots could contribute to insulin resistance: $1^{0}$ by altering supply (either in absolute terms or in particular anatomical locations, eg, portal vein to liver) or type of fatty acids, which then impact on insulin signaling or glucose metabolism in insulin-responsive tissues; $2^{0}$ by increasing or decreasing secretion of humoral factors from adipocytes (adipokines) or liver (hepatokines) that enhance (eg, adiponectin, fibroblast growth factor 21) or inhibit (eg, Fetuin A) insulin signaling; $3^{0}$ by attracting the invasion of cells, which release inflammatory cytokines (TNF) that impact on the insulin-signaling pathway in adjacent or distant tissue; $4^{0}$ sequestering a favorable factor (25-hydroxyvitamin D [25OHD]) or by conversion of a circulating factor to a form with more adverse metabolic activity (eg, conversion of cortisone

Citation: NGAYIMBESHA A, BIZIMANA JB, GAKIMA MS (2019) Effect of Eight Weeks of Exercise Training on Lipid Profile and Insulin Sensitivity in Obese Person. Int J Sports Exerc Med 5:119. doi. org/10.23937/2469-5718/1510119

Accepted: February 09, 2019; Published: February 11, 2019

Copyright: (c) 2019 NGAYIMBESHA A, et al. This is an open-access article distributed under the terms of the Creative Commons Attribution License, which permits unrestricted use, distribution, and reproduction in any medium, provided the original author and source are credited. 
to cortisol by 11 -hydroxysteroid dehydrogenase ; $5^{0}$ lipid depot volume could simply be a marker of a process that adversely affects insulin action.

Lifestyle factors such as sedentary way of life, overeating, alcohol consumption and others play a significant effect on insulin resistance development and also in the increase of lipids in the body. Weight loss through diet and exercise leads to significant health improvement and is, therefore, the key in treatment of obesity and insulin resistance related comorbidities [6].

Regarding exercise as a useful tool for obesity and insulin resistance management, most studies agree that both short time exercise and longtime exercise training improve body composition and together with improvements in insulin resistance [7]. However, there is a lack of consistency through studies results. One study affirmed that after 5 mouths of strength training program, there is no significant changes in fasting glucose and insulin resistance [8]. Another study investigated effects of aerobic training and resistance training on abdominal fat, intrahepatic lipid, and insulin sensitivity in obese adolescent males. In this study finding, no weight loss was observed but the results significant improvement in insulin sensitivity in the resistance training group. Also, a significant reduction in abdominal subcutaneous, visceral fat, and intrahepatic lipid percent in both the aerobic and resistance training group was observed. This study concluded that resistance training, independent of changes in diet or body weight, may be the most effective exercise modality to improve insulin sensitivity in obese adolescent males [9]. According this study, aerobic exercise does not positively influence insulin sensitivity. The present study aimed to assess the influence of a relatively low time of exercise training (8 weeks) on body lipids and insulin sensitivity in overweight/ obese person.

\section{Materials and Methods}

\section{Subject}

35 obese people who subscribed in fitness center for training and body weight control have been recruited to participate to the present study. Among participant, 8 were women and 27 men. Age $39 \pm 5$ years old; Weight $89 \pm 9 \mathrm{~kg}$ and Height $160 \pm 4 \mathrm{~cm}$. Inclusion criteria were: (1) To be able to brisk walk without assistance; (2) Without visual or hearing Impairments; to possess a $\mathrm{BMI}>30$ and who gives a written consent to participate in exercise program and accept all testing protocols. Excluded were those with complicating conditions (i.e., stroke, Parkinson's disease, renal failure, cardiac failure, pulmonary emphysema, or thyrotoxicosis). Prior to the study, all participants were given detailed information on the aim of the study, the process of the study and the time of experimentation. A written consent was obtained from every subject and/or his parent. Participants in the present study were not involved in regular physical exercise, but they showed their willingness to begin and finish the all physical program set by the study. Subjects visited physician first, for different examinations purpose. They were examined in terms of health status, history of disease and cardiorespiratory fitness in order to be sure that they can safely participate in the training program.

\section{Protocol of exercise training}

In this study, participants performed various aerobic exercises for 8 weeks, four sessions per week: Monday, Wednesday, Thursday and Friday. The duration of each session was predetermined: 2 hours in the morning and 2 hours in the afternoon. Exercising intensity was also predetermined $60 \%$ maximum heart rate.

The training shame was as follow:

Monday (From 8 a.m to 10 a.m): ball game such as basketball, football.

(From 2 p.m to 4 p.m): running on treadmill.

Wednesday (From 8 a.m to 10 a.m): running around football playground

(From 2 p.m to 4 p.m): Swimming in the swimming pool

Thursday (From 8 a.m to 10 a.m): ball game such as basketball, football.

(From 2 p.m to 4 p.m): running on treadmill.

Friday (From 8 a.m to 10 a.m): running around football playground.

(From 2 p.m to 4 p.m): Swimming in the swimming pool.

Each session exercise was constituted by three phases: warm-up, main exercise and cooling down part. The warm-up phase has taken 35 min to 40 minutes; it was constituted by jogging, slowly running, stretching exercise.

The main basic exercise has taken 1 hour and was constituted by physical training involving various aerobic exercises. The last part (cooling down) was performed by soft and slow running and stretching. It has taken about 20 minutes.

For the session on treadmill, after warming up, participant went on incline and motorized treadmill. Exercise intensity on treadmill was set at $6 \mathrm{~km} / \mathrm{h}$ to allow participant to accomplish running task without big difficult. Basketball session was constituted by actions such as dribbling, face to face ball pass and shooting. Another ball game used in our training program was Football. In this part of our training program, we mainly explored technical skills aspect in Football game. Examples of these skills developed are: ball control skills without opponent, ball control skills when under pressure of fatigue and ball control skills under threat from the opponent. Technical skills when moving were 
also explored. These technical skills included: -taking the ball and controlling the first pass; - dribbles, feints, various type of crosses; -follow-up (head, feet) and finishing; -one or two touches and pass and making the light choice quickly.

Each subject wore a Polar Heart Rate Monitor while performing proposed exercise. Whether the heart rate during exercise reached the target heart rate was verified using this Polar Heart Rate Monitor (Polar S610i, Polar Electro, Finland).

\section{Measurements}

For biochemical analysis, three stages of bloodletting were performed: prior to exercise (week zero), fourth week and eighth week. In the first stage of bloodletting, subjects were recommended to do not any physical exercise two days before the test and maintain their habitual diet.

Blood lipid measurement: $5 \mathrm{ml}$ of blood was drawn after twelve hours of fasting from the left antecubital vein in sitting position at 8 a.m. Biochemical variables analyzed were Total Cholesterol (TC), Triglyceride (TG), Low Density Lipoprotein (LDL), High Density Lipoprotein (HDL), Very Low-Density Lipoprotein (VLDLP) and LowDensity Lipoproteins to High Density Lipoproteins Ration (LDL/HDL). The blood samples were immediately centrifuged and refrigerated and then transported in cold storage to the Central Testing laboratory and analyzed within 24 hours.

Enzymatic method with auto analyzer alpha $x$ device was used for measuring lipid with (E2HL-100) kits and 0.1 (mmol/d) sensitivity (Hitachi, Tokyo, Japan).

Insulin sensitivity measurement: Insulin sensitivity was reflected in the Oral Glucose Tolerance Test (OGTT). After an 8-14 h fast, $75 \mathrm{~g}$ of glucose was given orally, and blood was drawn for plasma glucose and insulin determinations at $30,60,90$, and $120 \mathrm{~min}$ [10]. This study used the trapezoidal method for pharmacokinetics modeling to calculate the Area Under the Curve (AUC) for the 2-h integrated plasma glucose and insulin response to oral glucose challenge, which represents insulin sensitivity at different time points during the OGTT [11]. A lower AUC of glucose and insulin level reflects higher insulin sensitivity. Besides, a Homeostatic Model Assessment-Insulin Resistance (HOMA-IR) index was evaluated. A lower HOMA-IR reflects a lower insulin resistance and also higher insulin sensitivity [7].
Plasma glucose level was determined by a YSI model 2300 glucose analyzer (Yellow Springs Instrument Company, USA). Another glucose analyzer (YSI model 1500 glucose analyzer, Yellow Springs Instrument Company, USA) was used simultaneously to confirm validity. The correlation coefficient between the two analyzers was 0.90 based on the Pearson product-moment correlation analysis. Plasma insulin concentration was measured by radioimmunoassay with a coefficient of variation of $0.2 \%$.

\section{Statistical analysis}

Obtained results have been analyzed using SPSS software (vision 18). For lipid profile, variance with repeated measures has been performed to determine changes within the three stages of the test. T-test has also been performed to compare results after each test to the baseline. Significance of result was set at $P \leq 0.05$. For insulin sensitivity results, only means and standard deviation have been calculated.

\section{Ethics Consideration}

The ethic committee of the faculty of medicine within the Burundi University approved the study method and protocol. Our research procedure followed the Helsinki Declaration. All participants were assured that their participation was entirely voluntary and that they could withdraw at any time. Qualified subjects were admitted participating to the study upon their written consent.

\section{Results}

After 8 weeks of aerobic exercise training, an observable improvement of lipid profile in our participant has been obtained. In fact, a discernable decrease was observed in triglyceride, total cholesterol and lowdensity lipoprotein cholesterol. Also, an increase in high density lipoprotein cholesterol has been obtained after the completion of the training program. Below Table 1 shows statistical results of lipids after training program.

Statistical results contained in below Table 2 shows that all physiological indices tested: fasting blood glucose level, Oral Glucose Tolerance Test (OGTT), glucose Under Area Curve (UAC) and HOMA-IR decreased over time as the exercise training increase.

\section{Discussion}

After the completion of the prepared training program, selected lipid profile indicators have improved.

Table 1: Changes in lipid variables after 8 weeks of aerobic exercise training.

\begin{tabular}{|l|l|l|l|l|}
\hline Variables & Baseline & Fourth week & Eighth week & P Value \\
\hline TG $(\mathrm{mg} / \mathrm{dl})$ & $97.6 \pm 3.02$ & $97.4 \pm 2.4$ & $96.4 \pm 3.2$ & 0.001 \\
\hline TC $(\mathrm{mg} / \mathrm{dl})$ & $169 \pm 23$ & $168.7 \pm 16$ & $161.2 \pm 19$ & 0.001 \\
\hline LDL $(\mathrm{mg} / \mathrm{dl})$ & $112 \pm 17$ & $111.2 \pm 13$ & $107.9 \pm 15$ & 0.002 \\
\hline HDL $(\mathrm{mg} / \mathrm{dl})$ & $38.2 \pm 5.6$ & $38.9 \pm 3.7$ & $41.1 \pm 2.9$ & 0.001 \\
\hline VLDL $(\mathrm{mg} / \mathrm{dl})$ & $42.3 \pm 3.1$ & $41.9 \pm 2.6$ & $37.9 \pm 1.7$ & 0.001 \\
\hline HDL/LDL & $2.93 \pm 1$ & $3.12 \pm 1.2$ & $3.09 \pm 1.2$ & 0.001 \\
\hline TG A/HDL $(\mathrm{mg} / \mathrm{dl})$ & $2.55 \pm 1$ & $2.34 \pm 1.2$ & $2.33 \pm 1.2$ & 0.001 \\
\hline
\end{tabular}


Table 2: Change in glucose and insulin sensitivity after 8 weeks of training program.

\begin{tabular}{|l|l|l|l|l|}
\hline Variables & Baseline & \multicolumn{1}{l|}{ Fourth week } & \multicolumn{1}{l|}{ Eight weeks } & \\
\hline OGTT glucose level $(\mathrm{min})$ & & & & \\
\hline 0 & $8.4 \pm 2.9$ & $7.2 \pm 2.5$ & $7.1 \pm 1.8$ & 0.029 \\
\hline 30 & $12.7 \pm 4.0$ & $11.8 \pm 3.6$ & $11.4 \pm 2.5$ & 0.333 \\
\hline 60 & $15.6 \pm 3.6$ & $14.2 \pm 2.9$ & $13.3 \pm 2.9$ & 0.019 \\
\hline 90 & $17.9 \pm 4.3$ & $16.0 \pm 4.0$ & $15.4 \pm 3.6$ & 0.021 \\
\hline 120 & $18.6 \pm 6.1$ & $14.8 \pm 4.3$ & $14.7 \pm 4.3$ & 0.281 \\
\hline OGTT insulin level (min) & & & & 0.123 \\
\hline 0 & $41 \pm 43$ & $43 \pm 57$ & $10 \pm 18$ & 0.212 \\
\hline 30 & $73 \pm 79$ & $89 \pm 101$ & $81 \pm 108$ & 0.002 \\
\hline 60 & $159 \pm 133$ & $135 \pm 129$ & $121 \pm 133$ & 0.314 \\
\hline 90 & $166 \pm 151$ & $140 \pm 144$ & $156 \pm 191$ & 0.000 \\
\hline 120 & $152 \pm 144$ & $150 \pm 183$ & $107 \pm 191$ & 0.101 \\
\hline OGTT glucose AUC & $1915 \pm 449$ & $1605 \pm 317$ & $11631 \pm 13305$ & 0.023 \\
\hline OGTT insulin AUC & $14426 \pm 10800$ & $13307 \pm 13320$ & $0.5 \pm 0.2$ & 0.001 \\
\hline HOMA-IR & $2.4 \pm 0.8$ & $2.3 \pm 0.9$ & \\
\hline
\end{tabular}

First, LDL-C and HDL-C improved after training program. What is critical for the good health is not only the rise amount of cholesterol in the blood, but how it is distributed in different lipoprotein fractions is also equally important.

Raised concentration of plasma LDL-C and a low concentration of HDL-C fractions associated with high blood presence are the important risk factors of coronary heart disease. Low density lipoprotein constitutes the major transport form of cholesterol in the blood, which carry cholesterol from the liver to the various parts of body. An excess of cholesterol gets deposited in the arteries hence LDL-C is commonly known as bad cholesterol.

Our training program has affected Low Density Lipoprotein cholesterol. This lipid variable has decreased after 8 weeks of exercise training.

According to study conducted by Olson and colleagues in 2007, low-density lipoprotein and cholesterol are strongly affected by exercise [12]. In aerobic exercise, fat is used as the main source of energy. The decrease of LDL level in the blood after this training period can be explained by the effect of aerobic exercise on fat. In fact, during this type of physical exercise, fat is continuously broken down to produce energy required by the physical activity.

Wong and colleagues explain that physical activity, especially aerobic exercise increase lipoprotein lipase enzyme activity, and Lecithin Cholesterol Acyltransferase (LCAT) [13].

These two enzymes decrease triglyceride, LDL-C, and cholesterol while increase HDL-C. In addition, aerobic exercise absorbs cholesterol, decrease LDL, and subsequently prevent cardiovascular disease [14].

In literature, other researchers found results different to ours. In fact, using intense physical conditioning, Smoak, et al. observed no change in lipid profile among participant to his study [15]. This difference may be attributed to type of training different (aerobic exercise $><$ intense exercise) or to difference of participant body constitution.

Regarding High Density Lipoprotein Cholesterol (HDL-C), we observed an increase tendency of this lipid after the set training program. This increase tendency of HDL-C has also been observed by Archana Singh and Arti Sankhla [16]. The plausible explanation of HDL-C increase after our training program begins using fat during aerobic exercise.

This increase in HDL-C may be due to increased enzymatic activity (LPL) of lipoprotein lipase. LPL enzyme effectively converted VLDL to HDL. Increased activity of LPL enzyme increased levels of HDL-C. Furthermore, Lecithin Cholesterol Acyltransferase (LCAT) converts LDL cholesterol to HDL particles. Increasing this enzyme may be responsible for increasing HDL as a result of exercise.

Another possible reason for the increase in HDL may be due to increased HDL production by the liver followed by a change in (LPL) enzymatic activity and decrease in hepatic lipase followed by physical activity.

In the present study results, level of triglycerides in blood lowed after 8 weeks of aerobic exercise training. Triglycerides are the main source of energy during aerobic exercise, and aerobic exercises are known to increase the enzymatic activity of LPL. This enzyme is involved in fat metabolism. With the increase in this enzyme activity, the uptake of blood triglyceride is also increased. In other word, the increase of lipoprotein lipase as result of aerobic activity decreases triglyceride level in the blood.

The present study results showed a significant decrease of triglyceride to High Density Lipoprotein Ration (TG/HDL). This TG/HDL is known as a risk factor for cardiovascular disease. This ratio depends on HDL and cholesterol levels. Using a well-planned aerobic exercise, one of its results is the decrease of cholesterol level and the increase of HDL. The direct consequence of 
this change is the reduction of TG/HDL ration. Alhassan, et al. affirmed that triglyceride to high density lipoprotein ration constitute a relatively new lipoprotein index which can be used to predict the risk of heart diseases [17]. The ratio of TG/HDL represents the relative size of LDL particles and predicts athrogenicity. Low TG/HDL ratio represents large sized and non-atherogenic LDL while high TG/HDL ratio represents small sized, dense and atherogenic LDL particles. In our study, results showed that metabolic indicator declined. It means that regular physical training creates a favorable condition to decrease this ration, and as consequence to reduce its health threatens. The ration of LDL to HDL constitutes another cardiovascular risk factor. The highest ratio of LDL to HDL increases the risk of cardiovascular events and threatens individual health. Similar to TG/HDL, wellplanned aerobic exercise produces a reduction of $L D L$ to HDL ration.

The second and main aim of the present study was to determine the influence of aerobic exercise on glucose metabolism and insulin action improvement. Obtained results showed that our training program has positively influenced glucose uptake and insulin action. Our study results are in accordance with another study result's conducted by Jing Li and colleagues [18]. In their study titled "Duration of exercise as a key determinant of improvement in insulin sensitivity in type 2 diabetes", they observed an increased glucose uptake and insulin action improvement even 15 days after exercise training cessation. The improvement of insulin sensitivity was found through a decrease of OGTT, glucose AUC and HOMA-IR. However, in the present study subjects were obese person recruited in fitness center.

No diagnosis of diabetes or hyperinsulinemia was operated prior to exercise training. But, as our participants to the study were obese, we can speculate that they were in disposition to contact hyperinsulinemia or other metabolic problem. Hence, we can argue that 8 eight weeks of aerobic training improve insulin action in persons with metabolic abnormality such as type 2 diabetes patients or in situation of hyperinsulinemia.

Data lack to explain the process by which insulin sensitivity increase after exercise training. The increased insulin sensitivity after exercise training could be due to increased receptor affinity for insulin.

Our study has evaluated the trend of insulin at $30 \mathrm{~min}, 60 \mathrm{~min}$ and $120 \mathrm{~min}$ after exercise training cessation. Statistical results showed a decreasing tendency at each time. Even though we take a relatively short time, obtained insulin tendency confirms previous study results on insulin tendency after exercise training cessation. In fact, one study demonstrated retention of training related improvement in insulin action after 10 days without physical activity in master athletes [19].

Another study conducted by Dela, et al. reported a training induced increase in insulin action that persisted after 6 days of inactivity in overweight subjects [20]. Researchers have attempted to explain mechanism by which exercise training improves insulin action. One of explanation is that skeletal muscle mitochondrial content may constitutes an important factor governing insulin action [21]. Higher volume of exercise training (example more weekly training sessions) may induce changes in skeletal muscle mitochondrial content compared with low volume exercise training regimens [22]. The present study results confirm also results and conclusions of chia Huei Lin and colleagues [23]. In their study, they concluded that moderate intensity exercise training improves insulin sensitivity, quality of life and depression status in type 2 diabetes mellitus patients.

\section{Conclusion}

Eight weeks of aerobic exercise training improves body lipids profile. The same training program improves body glucose utilization and insulin sensitivity. Hence, well planned aerobic exercise considerably reduces metabolic problems including insulin sensitivity and other cardiovascular disease.

\section{Study Limitation}

The present study compared baseline results (before training program) to results after exercise training. Conclusion on exercise intervention effect will be more significant when data of control group was used rather than baseline results.

\section{References}

1. Jung UJ, Choi MS (2014) Obesity and its metabolic complications: The role of adipokines and the relation of obesity, inflammation, insulin resistance, dyslipidemia and non-alcoholic fatty disease. Int J Mol Sci 15: 6184-6223.

2. Saleh J, Sniderman AD, Cianflone K (1999) Regulation of Plasma fatty acid metabolism. Clin Chim Acta 286: 163-180.

3. Chiarelli F, Marcovecchio ML (2008) Insulin resistance and obesity in childhood. Eur J Endocrinol 159: S67-S74.

4. Ginsberg HN, Zhang YL, Hernandez-Ono A (2005) Regulation of plasma triglycerides in insulin resistance and diabetes. Arch Med Res 36: 232-240.

5. Krauss RM (1998) Triglycerides and atherogenic lipoproteins: rationale for lipid management. Am J Med 105: 58-62.

6. World Health Organization (2008) 2008-2013 Action Plan for the Global Strategy for the Prevention and Control of Noncommunicable Diseases; WHO Press: Geneva, Switzerland.

7. Matthews DR, Hosker JP, Rudenski AS, Naylor BA, Treacher DF, et al. (1985) Homeostasis model assesment: insulin resistance and $ß$-cell function from fasting plasma glucose and insulin concentrations in man. Diabetologia 28: 412-419.

8. Treuth M S, Hunter GR, Figueroa Colon R, Goran MI (1998) Effects of strength training on intra-abdominal adipose tissue in obese prepubertal girls. Medicine and Science in Sports and Exercise 30: 1738-1743.

9. Lee S, Fida B, Tamara H, Jennifer L Kuk, Chris Boesch, 
et al. (2012) Effects of aerobic versus resistance exercise without caloric restriction on abdominal fat, intrahepatic lipid, and insulin sensitivity in obese adolescent boys a randomized, controlled trial. Diabetes 61: 2787-2795.

10. Allison DB, Paultre F, Maggio C, Mezzitis N, Pi-Sunyer FX (1995) The use of areas under curves in diabetes research. Diabetes Care 18: 245-250.

11. Breda E, Cavaghan MK, Toffolo G, Polonsky KS, Cobelli C (2001) Oral glucose tolerance test minimal model indexes of beta-cell function and insulin sensitivity. Diabetes 50 : 150-158.

12. Olson TP, Dengel DR, Leon AS, Schmitz KH (2007) Changes in inflammatory biomerkers following one year of moderate resistance training in overweight women. Int $\mathrm{J}$ Obes 31: 996-1003.

13. Wong PC, Chai YH, Tson YH, Darren L (2008) Effects of 8 weeks of exercise training program on aerobic fitness, body composition, blood lipid and C-reactive protein in adolescents with obesity. Ann Acad Med Singapore 37: 86-93.

14. Wilund KR, Feeney LA, Tomayko EP, Weiss EP, Hagberg JM (2009) Effect of endurance exercise training on markers of cholesterol absorption and synthesis. Physiol Res 58: 545-552.

15. Smoak BL, Norton JP, Ferguson EW, Deuster PA (1990) Changes in lipoprotein profiles during intense military training. J Am Coll Nutr 9: 567-572.

16. Archana Singh, Arti Sankhla (2015) Aerobic Exercise Improves Lipid Levels of Normal and Obese Subjects. Int $J$ Pure App Biosci 3: 265-270.
17. Alhassan S, Kiazand A, Balise RR, King AC, Reaven GM, et al. (2008) Metabolic syndrome: do clinical criteria identify similar individuals among overweight premenopausal women? Metabolism 57: 49-56.

18. Li J, Zhang W, Guo Q, Liu X, Zhang Q, et al. (2012) Duration of exercise as a key determinant of improvement in insulin sensitivity in type 2 diabetes patients. Tohoku $\mathrm{J}$ Exp Med 227: 289-296.

19. Rogers MA, King DS, Hagberg JM, Ehsani AA, Holloszy JO (1990) Effect of 10 days of physical inactivity on glucose tolerance in master athletes. J Appl Physiol (1985) 68: 1833-1837.

20. Dela F, Larsen JJ, Mikines KJ, Ploug T, Petersen LN, et al. (1995) Insulin-stimulated muscle glucose clearance in patient with NIDDM. Effects of one-legged physical training. Diabetes 44: 1010-1020.

21. Bruce CR, Anderson MJ, Carey AL, Newman DG, Bonen A, et al. (2003) Muscle oxidative capacity is a better predictor of insulin sensitivity than lipid status. J Clin Endocrinol Metab 88: 5444-5451.

22. Bajpeyi S, Tanner CJ, Slentz CA, Duscha BD, McCartney JS, et al. (2009) Effect of exercise intensity and volume on persistence of insulin sensitivity during training cessation. J Appl Physiol 106: 1079-1085.

23. Chia-Huei Lin, Cheng WH, Liang CC, Cheng CC, Yi WW, et al. (2017) Effects of a 12-week Exercise Training on Insulin Sensitivity, Quality of Life, and Depression Status in Patients with Type 2 Diabetes 37: 227-236. 